\title{
Miniature ureteroscope distal tip designs for potential use in thulium fiber laser lithotripsy
}

Christopher R. Wilson

Joshua D. Kennedy

Pierce B. Irby

Nathaniel M. Fried 


\title{
Miniature ureteroscope distal tip designs for potential use in thulium fiber laser lithotripsy
}

\author{
Christopher R. Wilson, ${ }^{a}$ Joshua D. Kennedy, ${ }^{a}$ Pierce B. Irby, ${ }^{b}$ and Nathaniel M. Fried ${ }^{a, b, *}$ \\ aUniversity of North Carolina at Charlotte, Department of Physics and Optical Science, Charlotte, North Carolina, United States \\ ${ }^{b}$ Carolinas Medical Center, McKay Department of Urology, Charlotte, North Carolina, United States
}

\begin{abstract}
Thulium fiber laser lithotripsy using smaller optical fibers may enable development of miniature ureteroscopes. Two ureteroscope distal tip prototypes were built and characterized. The first design was 4.5-French (Fr) [1.5-mm outer diameter (OD)], five channel tip, housing 200- $\mu \mathrm{m}$ inner diameter (ID) dedicated central channel for insertion of $100-\mu \mathrm{m}$ core fibers and four surrounding channels, each with $1.5 \mathrm{Fr}(510-\mu \mathrm{m}$ ID) for instrumentation, irrigation, imaging, and illumination, respectively. The second design was $6.0-\mathrm{Fr}(2.0-\mathrm{mm}$ $\mathrm{OD})$, three-dimensional printed tip with larger, hemispherical common working channel and separate detection port integrated with ring lighting. Standard instruments, including optical fibers, guidewires, and stone baskets, were inserted through working channels to demonstrate feasibility. Gravitational and manual pump-assisted saline irrigation rates were measured. Luminous intensity distribution curves (LIDCs) were modeled for both ring and conventional lighting designs. Imaging was conducted using 3000,6000, and 10,000 pixel, miniature, flexible endoscopes with 0.4-, 0.6-, and 0.9-mm OD, to differentiate between urinary stones and ureter wall, for potential clinical application. The multichannel ureteroscope tip with 1.5-Fr working channel yielded a gravitational saline flow rate of $3.9 \pm 0.2 \mathrm{~mL} / \mathrm{min}$ compared to $31.3 \pm 0.6 \mathrm{~mL} / \mathrm{min}$ for standard (3.6 Fr) ureteroscope channel. Manual, pump-assisted irrigation increased flow rate to $32.5 \pm 3.0 \mathrm{~mL} / \mathrm{min}$. The 6000 pixel, $0.6-\mathrm{mm}$ $\mathrm{OD}$, flexible endoscope provided a balance of clear differentiation between stones and ureter wall and sufficiently small OD. A ring lighting configuration provided more uniform illumination than conventional cross-lighting geometry as demonstrated by LIDCs. With further development, these miniature ureteroscope tip designs may be integrated into a fully functional ureteroscope to permit ureteral access with minimal trauma and improved patient safety and comfort. ๑ 2018 Society of Photo-Optical Instrumentation Engineers (SPIE) [DOI: 10.1117/1.JBO.23.7.076003]
\end{abstract}

Keywords: illumination; irrigation; laser; lithotripsy; miniature; thulium; ureteroscope.

Paper 180133RR received Mar. 5, 2018; accepted for publication Jun. 21, 2018; published online Jul. 6, 2018.

\section{Introduction}

\subsection{Conventional Flexible Ureteroscopy and Holmium:YAG Laser Lithotripsy}

For the past two decades, trends in minimally invasive surgery for stone disease, principally ureteroscopy, have gradually embraced the utility and necessity of smaller instrumentation. ${ }^{1}$ To support this surgical technique, changes in the design and technology of specialized ureteroscopes, as well as various miniaturized instruments and accessories, have evolved. Ureteroscopy, by definition, implies passage of the instrument through the intact channels and orifices of the lower male and female urinary tract. Contemporary designs are approaching, but have not yet reached, a size that permits passage of instruments without some degree of manipulation and at least minimal, if only temporary and reversible trauma to the anatomy involved. The need and utility for smaller ureteroscopes and accessories to support this surgery are therefore obvious.

It is generally accepted that the necessary size of an instrument or ureteroscope to introduce into the ureter without trauma, or need to dilate the ureter, is about 1.7- to 2.0-mm diameter or 5 to 6 French $(\mathrm{Fr})$. Evolution of miniaturized designs for this purpose has progressed slowly. The first-generation modern

*Address all correspondence to: Nathaniel M. Fried, E-mail: nmfried@ uncc.edu flexible ureteroscope, introduced by Olympus (Tokyo, Japan) around 1996, had a distal tip diameter of $2.5 \mathrm{~mm}(7.5 \mathrm{Fr})$ with outer shaft diameter of $3.3 \mathrm{~mm}(10 \mathrm{Fr})$. Twenty years later, in 2016, the smallest currently marketed flexible ureteroscope was introduced (Olympus URF-P6), advertising a beveled tip diameter of $5 \mathrm{Fr}(1.7 \mathrm{~mm})$, but the shaft proximal to the tip quickly graduates to $8 \mathrm{Fr}(2.7 \mathrm{~mm})$ outer diameter. ${ }^{2}$ Even this most modern and advanced device still does not reach the dimensions required for truly atraumatic surgical procedures.

\subsection{Experimental Thulium Fiber Laser Lithotripsy}

The experimental thulium fiber laser (TFL) is currently being studied as a potential alternative to the standard holmium: yttrium aluminum garnet (YAG) laser for lithotripsy. (It should be noted that this fiber laser technology is fundamentally different from the solid-state, thulium:YAG laser currently being used in urology primarily for treatment of benign prostatic hyperplasia.) The TFL's more uniform Gaussian spatial beam profile enables delivery of higher laser power through smaller (e.g., 50 - and $100-\mu \mathrm{m}$ core) optical fibers than typically used during holmium lithotripsy (e.g., 200- $\mu \mathrm{m}$ core or greater). ${ }^{3-5}$ The holmium laser multimode beam prohibits coupling of high laser powers into small fibers, without overfilling the input fiber 
core and launching light into the fiber cladding, thus increasing the probability of damage to the proximal fiber connector. Holmium laser beams typically have diameters of 275 to $500 \mu \mathrm{m},{ }^{6}$ larger than 150 - and $200-\mu \mathrm{m}$ core fibers that urologists would ideally wish to use during lithotripsy procedures requiring extreme ureteroscope flexion (e.g., in lower pole of the kidney). Furthermore, it is difficult to pass fibers $>270-\mu \mathrm{m}$ core [464- $\mu \mathrm{m}$ outer diameter (OD)] through a flexed ureteroscope while simultaneously maintaining the maximum deflection angle of the ureteroscope. A smaller fiber diameter permits greater deflection of the ureteroscope and increased irrigation through the standard 1.2-mm inner diameter (ID) working channel, resulting in improved visibility and safety. TFL lithotripsy using smaller fibers may thus enable a reduction in both the working channel and ureteroscope dimensions.

Previous reports have already described the reduction in size of ureteroscope instruments for use with the TFL, such as a 1.9-Fr $(0.633 \mathrm{~mm})$ integrated fiber and stone basket device. ${ }^{7}$ A next logical step is to explore whether the ureteroscope itself can be sufficiently reduced to enable a TFL lithotripsy procedure without ureteral dilation.

\subsection{Ureteroscope Designs}

Ureteroscopes used in the clinic often feature only a single working channel for instruments and irrigation, alongside dedicated illumination and imaging channels. However, recent studies have reported that a dedicated irrigation channel, separate from the primary working channel, yields increased irrigation rates over a comparable cross-sectional size, single channel design. ${ }^{8-10}$ Dual-channel flexible ureteroscopes have been tested in the clinic, motivating movement toward multiple channels. It is therefore a logical next step to introduce more working channels beyond irrigation, for simultaneous insertion of different ureteroscope tools (e.g., optical fibers, guidewires, and stone baskets). Furthermore, recent studies have demonstrated efficacy of miniature ureteroscopes for less traumatic lithotripsy procedures. ${ }^{11,12}$ Thus, our first miniature ureteroscope tip design will explore a multichannel configuration.

Current ureteroscopes are also limited by suboptimal illumination, which presents shadows and hot spots in images, made worse by stone dust during lithotripsy. Suboptimal illumination may decrease ablation efficiency, increase operation time, and compromise patient safety. For example, several previous studies have reported accidental ureter perforation from the nitinol wires on stone baskets during holmium laser lithotripsy, due in part to poor visibility. ${ }^{13-17}$

Current optical illumination methods in ureteroscopy utilize single or double illumination points on the front face of the ureteroscope. This point source illumination method provides a measured 45 deg of high intensity and a 70-deg total field of light, causing harsh shadows across stone and tissue surfaces, making differentiation between stones and soft tissues difficult to resolve. Furthermore, this geometry separates the illumination channel from the viewing channel, limiting lighting in the foreground.

In an effort to counter this effect, many ureteroscope designs incorporate cross lighting by overlapping two point sources with a high numerical aperture, eliminating harsh shadows in the horizontal direction, but not in the vertical direction. Although this technique provides better coverage than single point illumination, short working distances make uniform illumination challenging. Alternatively, surrounding the front element of the viewing lens with a ring light may provide more uniform illumination. Ring illumination has been used on a larger scale for thoracic surgical procedures. ${ }^{18}$ A ring lighting geometry will be explored in our second miniature ureteroscope tip design.

This study describes design, characterization, and preliminary performance testing of two miniature ureteroscope distal tips. The first, multichannel, ureteroscope tip design was chosen based on current conventional geometries and the availability of off-the-shelf components for ease of assembly, while the second ureteroscope tip design was chosen based on the next generation approach of additive manufacturing, specifically threedimensional (3-D) printing capabilities. We also wanted to compare a multichannel, dedicated instrumentation approach with circular channel versus a single, multipurpose, hemispherical channel approach with ring lighting, with regards to space limitations and saline irrigation rates.

\section{Methods}

\subsection{Prototype Miniature Multichannel Ureteroscope Tip Design}

A first-generation prototype ureteroscope tip of 10-mm length was constructed in the laboratory (Fig. 1) out of translucent amber polyimide tubing, housing five cylindrical channels: a $200-\mu \mathrm{m}$ ID central channel for small fiber insertion and four peripheral working channels each with $510-\mu \mathrm{m}$ ID for instrumentation, irrigation, imaging, and illumination, respectively. These individual channels were then enclosed within a 4.35-Fr (1.45-mm ID) and 4.5-Fr (1.50-mm OD) polyimide sheath.

\subsection{Three-Dimensional Printed Ureteroscope Tip with Ring Lighting Geometry}

A second-generation 3-D printed ureteroscope tip design incorporated a dedicated 1.6-Fr (0.53-mm ID) port for ring illumination and detection [Fig. 2(b)]. This design also provided a single 5.4-Fr (1.8-mm ID) working channel with hemispherical shape to eliminate the wasted space observed between channels in Fig. 1. The ureteroscope tip was limited to 6-mm length and 6 Fr (2.0-mm OD) based on the 3-D printer (Form 2, Formlabs, Somerville, Massachusetts) capabilities. Three-dimensional printing was performed using a Formlabs proprietary UV curing photopolymer/photoreactive resin material of black color (RSGPBK-04, FormLabs Inc.). The purpose of this design was twofold: uniformity of light distribution for enhanced image quality and reduction in overall diameter of the ureteroscope distal tip.

\subsection{Instrument Insertion}

Passage of a $100-\mu \mathrm{m}$ core, $170-\mu \mathrm{m}$ OD optical fiber (FIP100140170, Polymicro, Phoenix, Arizona) through a 200- $\mu \mathrm{m}$ ID central channel may free up valuable space that could be used to either decrease tip outer diameter or provide more space for larger working channels, while still remaining within the 5- to 6-Fr (1.7 to $2.0 \mathrm{~mm}$ ) size range targeted for the miniature ureteroscope. Hydrophilic guide wires are typically used to facilitate safe access into the upper urinary tract. Guide wire insertion was successfully conducted with a $450-\mu \mathrm{m}$ OD (0.018 in) hydrophilic guide wire (Terumo, Somerset, New Jersey) through one of the working channels. A 1.3-Fr (433- $\mu \mathrm{m}$ OD) stone basket (Optiflex Nitinol Stone Retrieval 

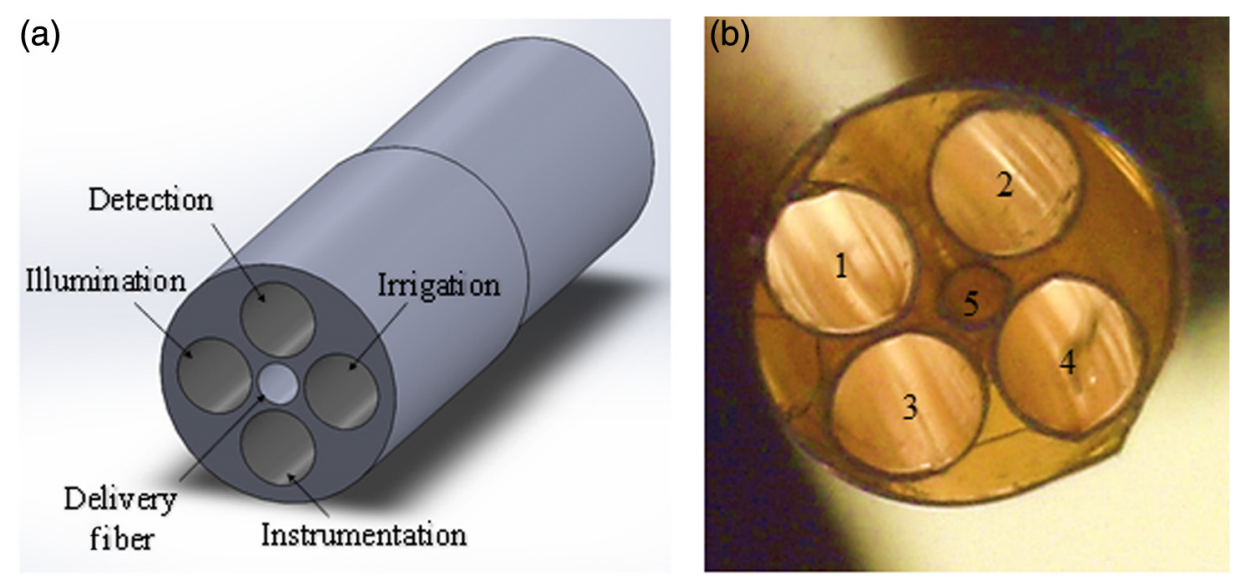

Fig. 1 (a) Diagram and (b) photograph of miniature, 10-mm-long, 4.5-Fr (1.5-mm OD) ureteroscope tip with five channels: (1) illumination, (2) detection, (3) guidewire/stone basket, (4) irrigation, and (5) laser fiber.
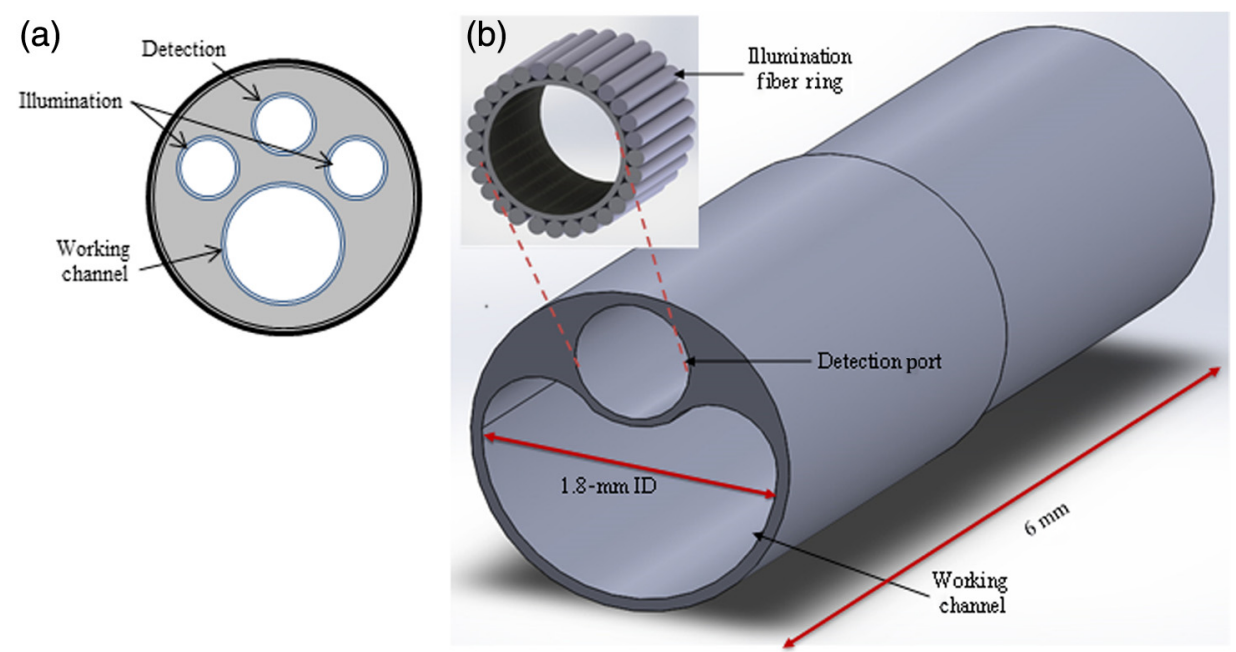

Fig. 2 (a) Diagram of conventional 8-Fr (2.7-mm OD) ureteroscope distal tip with 3.4-Fr (1.2-mm ID) working channel. (b) Diagram of 6-mm-long, 6-Fr (2.0-mm OD) miniature ureteroscope tip with dedicated 1.6-Fr (0.53-mm ID) port for illumination and detection, and single hemispherical 5.4-Fr (1.8-mm ID) working channel for irrigation, fiber, and guidewire/stone basket. Inset: illumination fiber distribution within channel.

Basket, Boston Scientific, Marlborough, Massachusetts) was also successfully inserted through a working channel of the multichannel ureteroscope tip (Fig. 3).

Similarly, for the second, 3-D printed ureteroscope tip design, passage of various ureteroscope instruments was conducted to demonstrate feasibility. Figure 4 shows the 6-Fr (2-mm OD) ureteroscope tip working channel with insertion of ureteroscope instrumentation and irrigation, including the empty channel, with 1.3 -Fr stone basket insertion, with $100-\mu \mathrm{m}$ core (170- $\mu \mathrm{m}$ OD) delivery fiber insertion, and with gravitational saline flow through the working channel.

\subsection{Prototype Testing_In Vitro Imaging}

A 0.4-mm OD, 600-mm-long, 3000 pixel endoscope (Milliscope II, Advanced Inspection Technologies, Melbourne, Florida) was tested with our prototype tips. This endoscope provided a 55-deg field of view, 8-mm bending radius, and 4- to 10-mm adjustable depth of field (Table 1). A standard 50-watt LED light source (LLS-050, AIT) was used with the endoscope for illumination [Fig. 5(a)].

Fresh ex vivo porcine ureters were detached from kidneys obtained from a local slaughterhouse and then sectioned into 7-cm-long samples and immersed in a saline bath to simulate the clinical environment for imaging through the ureteroscope tip. Ureters were kept hydrated in saline and the distal end of the ureter was clamped to prevent light from entering. Uric acid (UA), calcium oxalate monohydrate (COM), calcium oxalate dihydrate (COD), and 60\% COM/40\% calcium phosphate (CaP) samples, commonly encountered in the clinic, ${ }^{19}$ were placed in ureters for image testing (Fig. 6).

\subsection{Comparison of Miniature Endoscopes}

The 6000 and 10,000 pixel flexible fiber optic endoscopes (Myriad Fiber Imaging, Dudley, Massachusetts) were selected for comparison based on image quality (Table 1). These endoscopes, too large for insertion through the prototype 


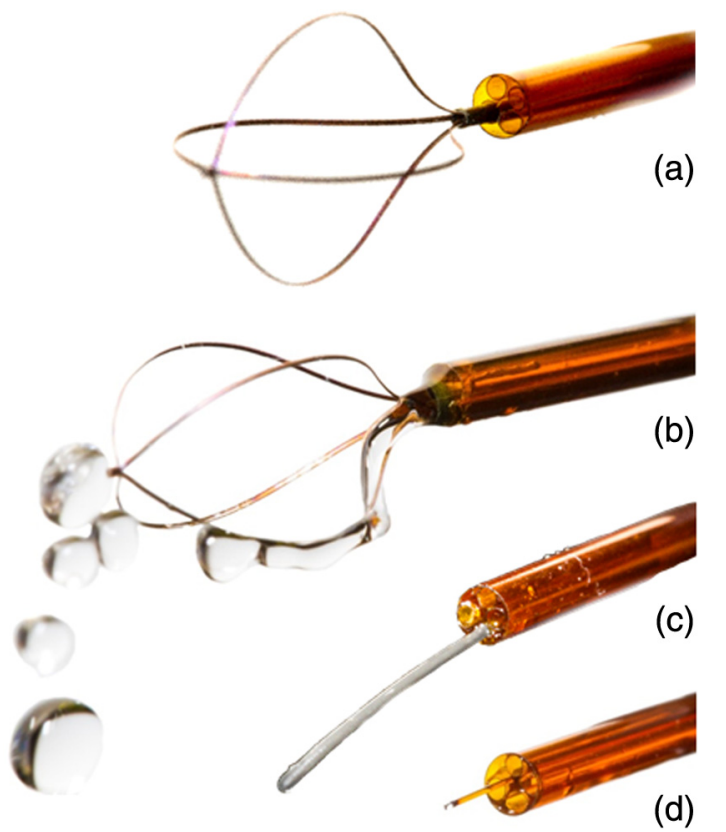

Fig. 3 Miniature ureteroscope tip with device demonstrations: (a) 1.3Fr stone basket insertion, (b) stone basket insertion with saline flow through separate channels, (c) guidewire insertion, and (d) $100-\mu \mathrm{m}$ core $(170-\mu \mathrm{m} \mathrm{OD})$ delivery fiber insertion through central channel.
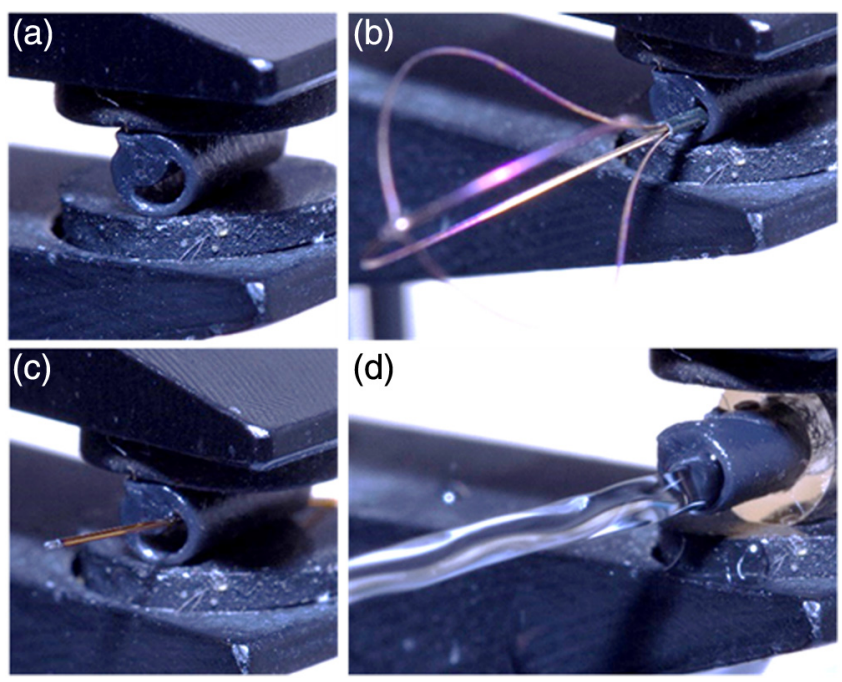

(d)

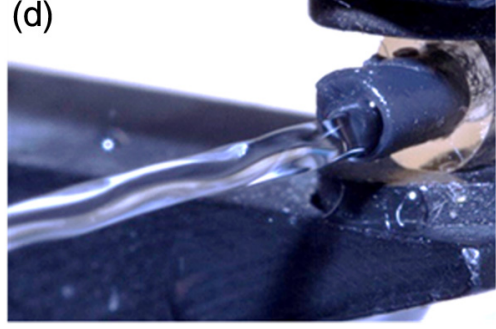

Fig. 4 Miniature, 3-D printed, 6-Fr (2-mm OD) ureteroscope tip working channel with insertion of ureteroscope instrumentation and irrigation: (a) prototype alone, (b) 1.3-Fr stone basket insertion, (c) $100-\mu \mathrm{m}$-core $(170-\mu \mathrm{m} \mathrm{OD})$ delivery fiber insertion, and (d) gravitational saline flow through working channel.

Table 1 Specifications of miniature endoscopes.

\begin{tabular}{lccc} 
Number of pixels & 3000 & 6000 & 10,000 \\
Field of view $(\mathrm{deg})$ & 55 & 65 & 70 \\
Working distance $(\mathrm{mm})$ & 4 to 10 & 3 to 12 & 3 to 15 \\
Outer diameter $(\mathrm{mm})$ & 0.4 & 0.6 & 0.9 \\
Bend radius $(\mathrm{mm})$ & 8 & 15 & 10 \\
\hline
\end{tabular}
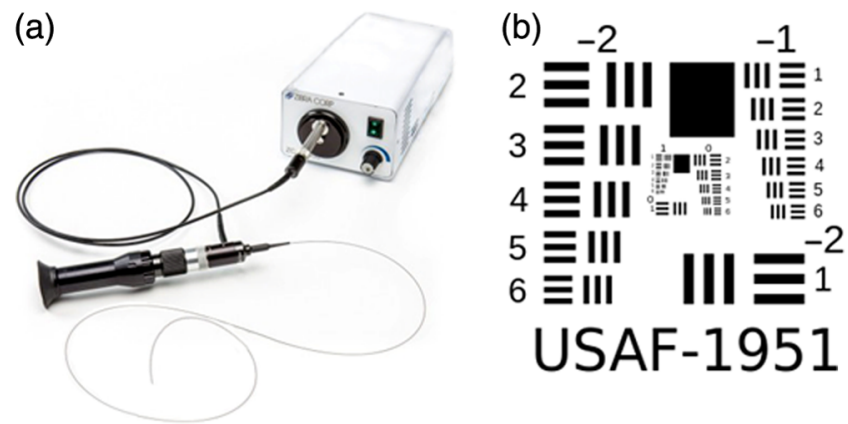

Fig. 5 (a) Miniature endoscope system, showing fiber optic bundle and light source. Fiber optic bundles with 0.4-, 0.6-, and 0.9-mm diameters corresponding to 3000,6000 , and 10,000 pixel resolution, respectively, were used in this study. (b) 1951 USAF resolution test chart used for miniature endoscope imaging comparison.

ureteroscope tip working channels, were instead operated freely in vitro in clamped tygon tubing submerged in saline. A standard United States Air Force (USAF) resolution test chart [Fig. 5(b)] was used to compare images from 3000, 6000, and 10,000 pixel endoscopes at working distances of 1 to $5 \mathrm{~mm}$.

\subsection{Saline Irrigation Rate Studies}

Saline irrigation rates were measured and compared to a standard flexible ureteroscope (Uretero-Reno Videoscope URF-V, Olympus, Southborough, Massachusetts) working channel with and without instruments (e.g., optical fiber or stone basket) present. Both ureteroscope tips were connected to tubing of 600-mm length and 2-mm ID. Irrigation rates were measured by introducing gravitational flow from a saline bag (at a fixed height of $100 \mathrm{~cm}$ ) for $2 \mathrm{~min}$ for each experiment. The ureteroscope tips and 600-mm length tubing were placed in a horizontal orientation, similar to a clinical configuration. A simple manual pump (Single Action Pumping System, Boston Scientific, Marlborough, Massachusetts), commonly used in ureteroscopy, consisting of 10-mL syringe, tubing, valve, and clamp was also tested for enhancing irrigation rates. The manual pump was pressure controlled, with flow proportional to the user's pressure from force exerted on the syringe, consistent with hand pumps currently used in the clinic. Studies were conducted using a combination of different fibers and stone baskets, including 100- $\mu \mathrm{m}$ core fiber (FIP100140170, Polymicro, Phoenix, Arizona), 270- $\mu \mathrm{m}$ core fiber (Holmium Lightguide 270D fiber, Olympus Gyrus ACMI), and 1.3-Fr stone basket (Optiflex Nitinol Stone Retrieval Basket, Boston Scientific, Natick, Massachusetts). Saline volume was measured with a graduated cylinder. A sample size of $n=3$ was performed for each study with mean \pm standard deviation of flow recorded and compared to flow through the empty working channel (control).

\subsection{Illumination Studies}

Luminous intensity is a measurement of light in terms of its perceived brightness to the human eye, from the power emitted by a light source. Luminous intensity distribution curves (LIDCs) are graphical representations of the luminous intensity in a plane passing through the source, demonstrating the luminosity of the source(s) as the light propagates in a forward direction. This is a standard method for quantifying lighting distributions 


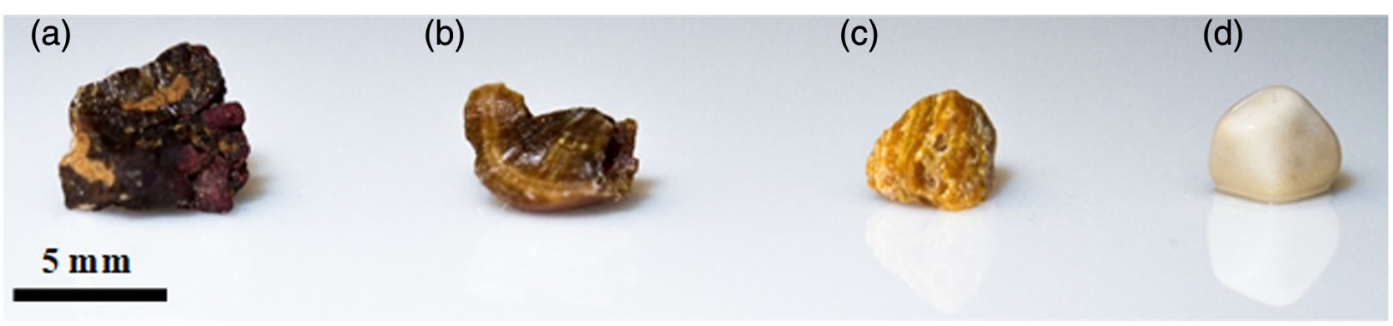

Fig. 6 Representative kidney stone compositions used in endoscope resolution comparison, showing (a) COM, (b) COD, (c) UA, and (d) CaP.

for different illumination configurations, which has previously been reported. ${ }^{18}$

The curve measurements were determined by tracing out individual fiber illumination cast over graph paper. Sequential fiber orientation illumination fields were traced over one another until a complete plot of fields yielded a coverage map or LIDC. This was repeated for both a conventional ureteroscope tip design with cross lighting [Fig. 2(a)] as well as our experimental ureteroscope tip design with ring lighting [Fig. 2(b)], and then transferred to a PowerPoint image.

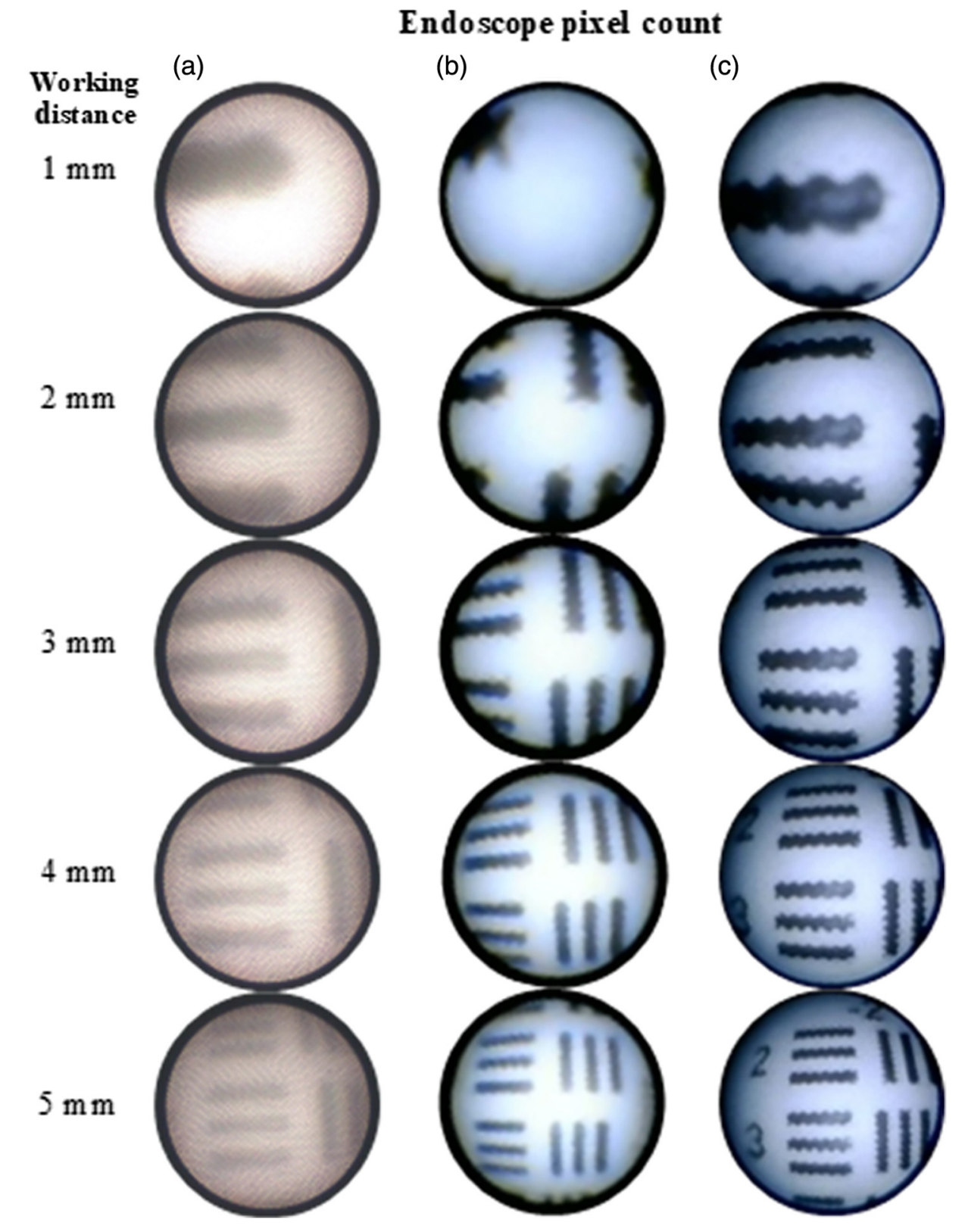

Fig. 7 Image quality comparison of (a) 3000, (b) 6000, and (c) 10,000 pixel endoscopes using the 1951 USAF resolution test chart. Working distances range from 1 to $5 \mathrm{~mm}$. 


\section{Results}

\subsection{Multichannel Ureteroscope Tip Configuration}

Calibration results using a USAF resolution test chart are shown in Fig. 7, for the three miniature endoscopes tested (3000, 6000, and 10,000 pixels). An in vitro porcine ureter model was also used to demonstrate sufficient image contrast between four different common urinary stone compositions (COM, COD, UA, and $\mathrm{CaP}$ ) and the ureter for each of the miniature endoscopes (Fig. 8). Sufficient image contrast was judged based on the point at which 1-to-1 imaging fibers were no longer distinguishable, but the object of interest (e.g., kidney stone) was distinguishable from its background (e.g., ureter wall). Comparison of the images demonstrated the 3000 pixel endoscope to be a viable, but poor option, as the imaging fiber footprint was translated to the output image, decreasing visibility as well as overall contrast, therefore rendering stones harder to differentiate from the ureter background. However, both 6000 and 10,000 pixel endoscopes provided improved image quality compared to the 3000 endoscope, as expected.

\subsection{Three-Dimensional Printed Ureteroscope Tip with Ring Lighting Geometry}

LIDCs are shown in Fig. 9 for both the conventional ureteroscope tip [Fig. 2(a)] and the experimental ureteroscope tip [Fig. 2(b)], demonstrating the ring illumination's uniformity of distribution. For both images, multiple red-dashed circles represent the field of illumination for individual fiber sources, while the blue inlaid region signifies overlap of multiple fields, gathered from sequential hand plotting of individual fiber sources. It is this overlapping region that is of most interest, demonstrating the uniformity of the lighting coverage. Figure 9 is a representative illustration of two illumination configurations, the conventional and the experimental designs, quantified (a)
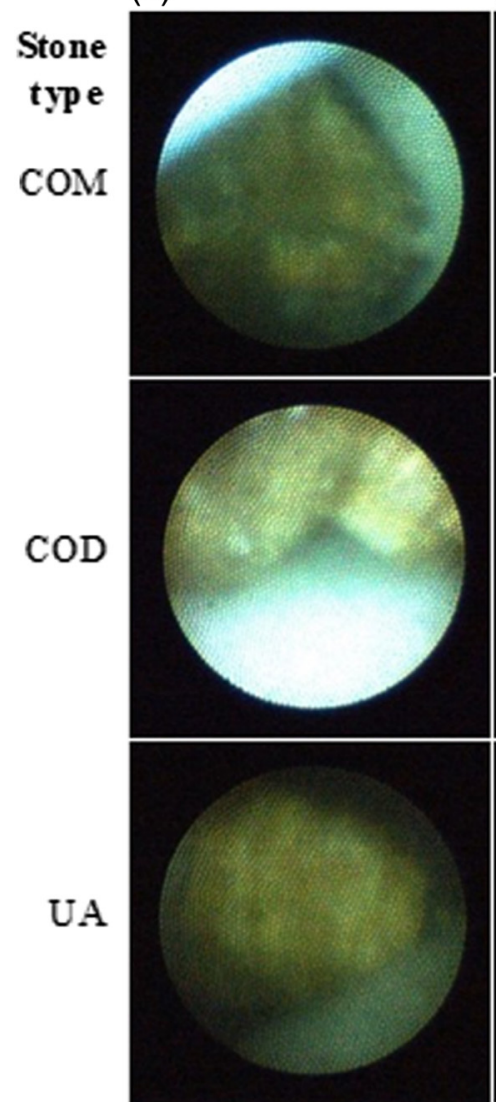

(b)

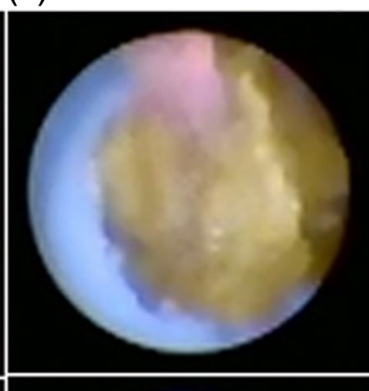

(c)
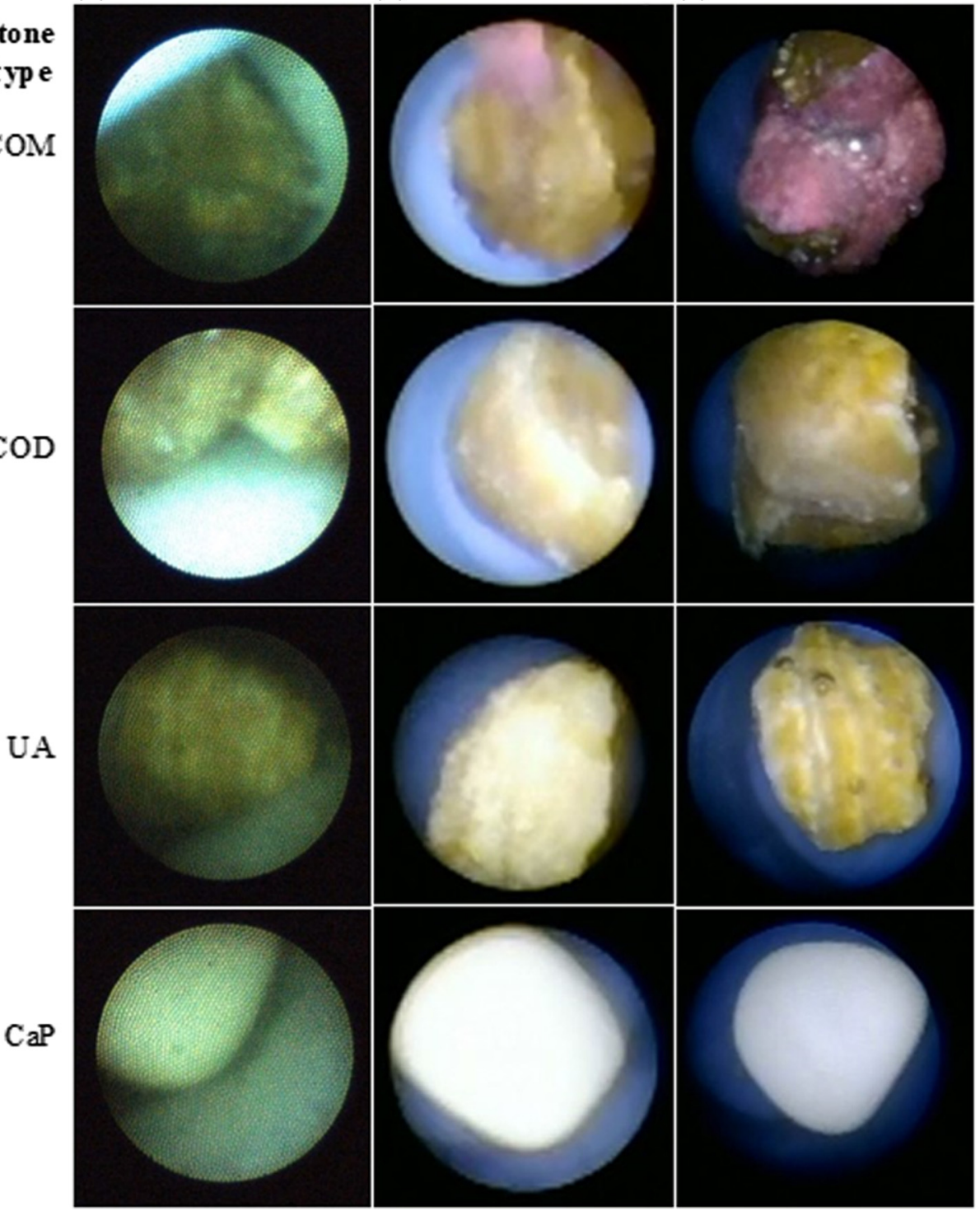

Fig. 8 In vitro image quality comparison of COM, COD, UA, and CaP stones with (a) 3000, (b) 6000, and (c) 10,000 pixel endoscopes. 


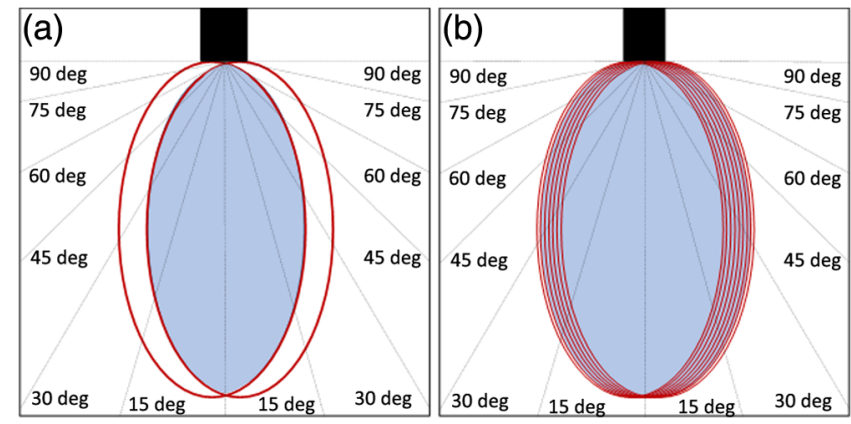

Fig. 9 LIDC, graphically representing the luminous intensity in a plane passing through the ureteroscope tip, as they propagate in a forward direction from the near, to the far field regime (0 to $14 \mathrm{~mm}$ ). (a) LIDC for conventional ureteroscope tip shown in Fig. 2(a). (b) LIDC for experimental ureteroscope tip design shown in Fig. 2(b), demonstrating ring illumination's uniformity of distribution. For both images, multiple red circles represent field of illumination for individual fiber sources, while blue inlaid region signifies overlap of multiple fields, gathered from sequential hand plotting of individual fiber sources. It is this overlapping region that is of most interest, demonstrating the uniformity of the lighting coverage.

by physically hand tracing the light from the fiber projected on graph paper. This representation is uniaxial, so while the ring illumination will remain constant when rotated at $90 \mathrm{deg}$, the illumination coverage with the conventional geometry will become unity, yielding a poor distribution and coverage.

Furthermore, as observed in the LIDCs in Fig. 9(a), the conventional illumination geometry for ureteroscopes is based on the cross-lighting principle, in which two sources overlap for even lighting centered in front of the viewing optic (blue region). The configuration utilizes each source as a fill light for the other, highlighting dark areas created by its partner (red outline). However, the illumination points shown are not in line with the detection port, casting shadows above the subject, and limiting visibility in the vertical direction.

Ring illumination softens shadows without eliminating necessary depth information. With the incorporation of high numerical aperture optical fibers, ring illumination is distributed over large angles, reducing glinting and hot spots. These hot spots may be further reduced by diffusing the light through polishing the ring lighting with a matte finish where the surface is rough on a microscopic scale. The LIDCs shown in Fig. 9 demonstrate the distribution difference in axially symmetric lighting geometry versus traditional dual-point source geometry. The overlapping field of even illumination is extended across the entire traditional uneven light field. The illumination falls outside the imaging field of view, which means that the experience of falloff will be less pronounced within the image, even filling in shadows behind the subject, for example, through reflection off the kidney or ureter wall.

\subsection{Saline Irrigation Rates}

Figure 10 plots the saline irrigation rates for both miniature ureteroscope designs with comparison to a standard ureteroscope working channel, with and without instruments (e.g., optical fiber or stone basket) present. When comparing gravitational flow saline irrigation rates for the empty standard working channel versus those of the ureteroscope tip prototypes, saline rates through a single $510-\mu \mathrm{m}$ port of the five-channel design were found to be insufficient. The decrease in inner diameter of the four channels, from 1.200 to $0.510 \mathrm{~mm}$, resulted in a decrease in flow rate from $31.3 \pm 0.6$ to $3.9 \pm 0.2 \mathrm{~mL} / \mathrm{min}$. However, manual pump-assisted irrigation yielded $32.5 \pm$ $3.0 \mathrm{~mL} / \mathrm{min}$, which may provide an alternative to conventional gravitational flow irrigation. The 3-D printed ureteroscope tip with a larger, 1.8-mm hemispherical working channel shape (cross-sectional area of 1.55 versus $1.13 \mathrm{~mm}^{2}$ ), surpassed

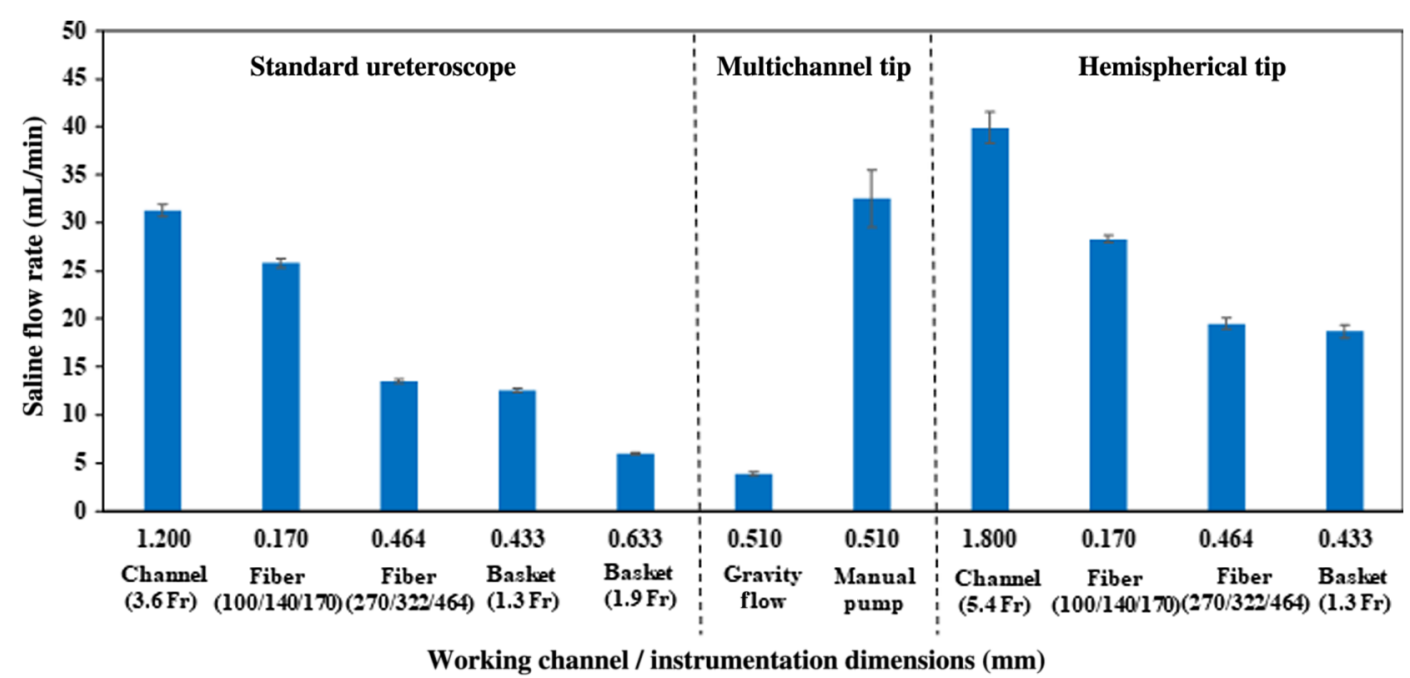

Fig. 10 Comparison of irrigation rates through 3.6- (1.2-mm ID), 1.5- (0.510-mm ID), and 5.4-Fr (1.8-mm ID hemisphere) working channels of standard and prototype ureteroscope tips, with and without instruments (e.g., optical fiber or stone basket) present. Both ureteroscope tips were connected to $600-\mathrm{mm}$ length and 2-mm ID tubing. Irrigation rates were measured utilizing gravitational flow from a saline bag at a height of $100 \mathrm{~cm}$ for $2 \mathrm{~min}$ for each study $(n=3)$. Ureteroscope tips and tubing were placed in a horizontal orientation to simulate a clinical environment. A manual pump consisting of $10-\mathrm{mL}$ syringe, tubing, valve, and clamp was also tested. The pump was pressure controlled, with flow proportional to user's pressure from force exerted on syringe. 
the standard flow rate by an additional $28 \%$, measuring $39.9 \pm 1.6 \mathrm{~mL} / \mathrm{min}$.

\section{Discussion}

\subsection{Multichannel Ureteroscope Tip}

The experimental, $100-\mu \mathrm{m}$ core, TFL optical fiber has a small cross-sectional area, thereby enabling an increase in the saline flow rate through a standard 1.2-mm ID working channel, as compared with the standard clinical holmium $270-\mu \mathrm{m}$ core fiber. As a result, such a large working channel may be unnecessary during TFL lithotripsy. In our five-channel prototype ureteroscope tip design, every instrument as well as irrigation flow had a dedicated channel. Although gravitational flow through the $0.510-\mu \mathrm{m}$ channel was insufficient, addition of a manual pump increased the flow rate by an order of magnitude. Such a proximal pumping mechanism, sometimes utilized in the clinic, would need to be integrated into the final ureteroscope tip design to provide suitable visibility and safety during laser lithotripsy. Automated saline irrigation systems have also recently been introduced and may provide another reliable alternative for use during ureteroscopy procedures. ${ }^{20}$

Since we have demonstrated the feasibility of a ureteroscope design with an outer diameter $<5 \mathrm{Fr}$, the working channels may be potentially redesigned with altered arrangements, diameters, and geometries within the same total area footprint, for desired functionality. Enlarging one of the $510-\mu \mathrm{m}$ working channels to $600 \mu \mathrm{m}$ in order to accommodate the diameter of a 6000 pixel integrated endoscope is one such option. In this case, since illumination is integrated into the endoscope, a dedicated illumination working channel could be removed, freeing up another $510 \mu \mathrm{m}$ of usable area. This flexibility in the working channel specifications may also allow multiple access points for and variability of the instruments themselves, such as maneuvering and substituting the fiber laser and stone basket for easier access, dependent on the stone's orientation within the ureter or kidney. As for the other, 3-D printed ureterscope tip, elimination of the protective sheath used for packaging the 6000 endoscope as a single system, would enable it to be accommodated within the 3-D printed detection port, since the 0.6-mm OD endoscope is already within tolerance of the $0.53-\mathrm{mm}$ port opening. The detection port could be adjusted to accommodate the minimal increase, without compromising the overall endoscope tip OD.

It should also be noted that although our preliminary ureteroscope tip designs did not incorporate wire cables, required for manipulation (e.g., steering and deflection) in any flexible ureteroscope, there is sufficient space for future addition of these wires. For example, as shown in Fig. 1, there is ample unused space in multiple places in between the five dedicated channels. Also, in Fig. 2, there is space on either side of the detection port for future attachment of wire cables in the 3-D printed design. This preliminary study focused on the design of ureteroscope distal tips, as opposed to the fabrication of a complete, functional endoscope, which will be the subject of future work. Future studies will also need to take into account potential changes in saline irrigation rates as a function of ureteroscope deflection.

\subsection{Three-Dimensional Printed Ureteroscope Tip}

Although the smaller, dedicated TFL fiber working channel in the multichannel ureteroscope tip design enabled higher saline irrigation rates, with the use of a manual pump, in comparison to use of a larger holmium fiber within a standard working channel, the 3-D printed ureteroscope tip yielded even higher saline irrigation rates, which should translate into further improvement in visibility and safety in the clinic. The hemispherical geometry may also provide added maneuverability of the instrumentation within the working channel. This initial prototype demonstrated the feasibility of additive manufacturing but was limited by the capabilities of a relatively inexpensive 3-D printer. Use of higher resolution 3-D printers may enable production of smaller ureteroscope tips down to 5 Fr (1.7-mm OD).

It should be noted that these design specifications should be considered in the context of current beveled tip ureteroscopes. While the beveling process allows for easier initial insertion of the ureteroscope tip, it does not eliminate the need to pass the thicker shaft through the same access point (e.g., ureter). Our miniature ureteroscope tip designs could in principle be successfully inserted though the ureter without the need for dilation, which would be a major advance in the minimally invasive technique. However, as noted above, it should also be emphasized that future miniature ureteroscope designs will also have to take into consideration the need for integrating an active flexion mechanism within the shaft of the ureteroscope to ensure access into the ureter.

\subsection{Illumination}

While initial studies have been conducted involving high numerical aperture optical fibers in a circular configuration, there is some inherent loss of potential illumination from interfiber dead space. Future work may evaluate custom-built plastic fibers and liquid light guides in the same ring orientation without the undesirable losses typically encountered in circular fiber bundling. Further studies may also explore diffuse lighting by altering the distal end of the illumination guide to enhance scattering, thus reducing the "hot spot" effect, which can effectively flatten an image by overexposing the foreground and decreasing depth perception.

Narrow band imaging (NBI), an optical image enhancement technology in which specific bandwidths are implemented, may also be deployed to increase image contrast and visibility between different tissues. NBI is currently used for bladder cancer, colonic polyps, and Barrett's esophagus cancer detection during cystoscopy, whereby illuminating light is in two specific wavelength bands, blue (440 to $460 \mathrm{~nm}$ ) and green (540 to $560 \mathrm{~nm})$, which are strongly absorbed by hemoglobin. ${ }^{21-25}$ Commercially available NBI systems consist of narrow band filters in front of a white light source ${ }^{26,27}$ and use of 415and 540-nm light sources. ${ }^{28}$ Application of NBI during laser lithotripsy procedures would focus on distinction between urinary stones and the ureter and kidney walls, rather than vascularization, to prevent perforation of the ureter, due in part to poor visibility during laser lithotripsy.

\section{Conclusions}

A 4.5-Fr, multichannel ureteroscope tip was designed, housing a dedicated $200-\mu \mathrm{m}$ ID central channel for small fibers and four surrounding channels, each with $510-\mu \mathrm{m}$ ID for instrumentation, irrigation, imaging, and illumination. Optical fibers, guidewires, and stone baskets were successfully inserted through these channels. Low gravitational saline irrigation rates necessitated the use of manual pump-assisted irrigation for potential clinical 
use. Imaging was performed with 3000,6000 , and 10,000 pixel flexible endoscopes with 0.4-, 0.6-, and 0.9-mm outer diameters, respectively. The 6000 pixel endoscope enabled an optimal balance of adequate illumination and differentiation between stone sample and surrounding ureter wall while also providing a sufficiently small outer diameter for future integration into a fully functional ureteroscope. The concept of a 6-Fr ureteroscope tip with integrated detection/ring lighting illumination port and larger single hemispherical working channel was also designed to provide more uniform illumination compared to conventional single and double point source geometries. With further development, these miniature ureteroscope tips may be integrated into a fully functional ureteroscope, permitting ureteral access without compromising performance.

\section{Disclosures}

Nathaniel M. Fried is a consultant with IPG Medical Corporation (Marlborough, Massachusetts), but he does not hold any financial stake in the company. None of the other authors have a financial interest in this paper.

\section{Acknowledgments}

Joshua D. Kennedy was supported by a summer undergraduate research fellowship from the Charlotte Research Scholars Program at UNC-Charlotte. Christopher R. Wilson was supported by a Lucille P. and Edward C. Giles Dissertation Year Graduate Fellowship Award from the Graduate School at UNC-Charlotte. This paper was based in part on the following SPIE conference proceedings papers: C. R. Wilson et al., "Novel ureteroscope illumination designs," Proc. SPIE 10038, 100380P (2017) and J. D. Kennedy et al., "Miniature ureteroscope tip designs for use in thulium fiber laser lithotripsy," Proc. SPIE 10038, 100380Q (2017).

\section{References}

1. S. G. Hubosky et al., "Accessing the difficult ureter and the importance of ureteroscope miniaturization: history is repeating itself," Urology 84(4), 740-742 (2014).

2. Olympus, URF-P6, Center Valley, Pennsylvania, http://medical .olympusamerica.com/products/ureteroscope/fiberscope-urf-p5.

3. N. J. Scott, C. M. Cilip, and N. M. Fried, "Thulium fiber laser ablation of urinary stones through small-core optical fibers," IEEE J. Sel. Top. Quantum Electron. 15(2), 435-440 (2009).

4. R. L. Blackmon, P. B. Irby, and N. M. Fried, "Thulium fiber laser lithotripsy using tapered fibers," Lasers Surg. Med. 42(1), 45-50 (2010).

5. R. L. Blackmon et al., "Thulium fiber laser ablation of kidney stones using a 50- $\mu$ m-core silica optical fiber," Opt. Eng. 54(1), 011004 (2015).

6. S. Griffin, "Fiber optics for destroying kidney stones," Biophotonics Int. 11(4), 44-47 (2004).

7. C. R. Wilson et al., "A miniaturized, 1.9F integrated optical fiber and stone basket for use in thulium fiber laser lithotripsy," J. Endourol. 29(10), 1110-1114 (2015).

8. T. Bach et al., "Working tools in flexible ureterorenoscopy-influence on flow and deflection: what does matter?" J. Endourol. 22(8), 1639-1643 (2008).

9. K. Haberman et al., "A dual-channel flexible ureteroscope: evaluation of deflection, flow, illumination, and optics," J. Endourol. 25(9), 1411-1414 (2011).

10. A. Lusch et al., "Comparison of optics and performance of single channel and a novel dual-channel fiberoptic ureteroscope," Urology 85(1), 268-272 (2015).
11. J. P. Caballero et al., "Micro-ureteroscopy: initial experience in the endoscopic treatment of pelvic ureteral lithiasis," Actas Urol. Esp. 39(5), 327-331 (2015).

12. J. P. Caballero-Romeu et al., "Assessment of the effectiveness, safety, and reproducibility of micro-ureteroscopy in the treatment of distal ureteral stones in women: a multicenter prospective study," J. Endourol. 30(11), 1185-1193 (2016).

13. R. W. Santa-Cruz, R. J. Leveillee, and A. Krongrad, "Ex vivo comparison of four lithotripters commonly used in the ureter: what does it take to perforate?" J. Endourol. 12(5), 417-422 (1998).

14. M. Sofer et al., "Holmium: YAG laser lithotripsy for upper urinary tract calculi in 598 patients," J. Urol. 167(1), 31-34 (2002).

15. A. M. Althunayan et al., "Adverse events resulting from lasers used in urology," J. Endourol. 28(2), 256-260 (2014).

16. J. Cordes et al., "Destruction of stone extraction basket during an in vitro lithotripsy-a comparison of four lithotripters," J. Endourol. 25(8), 1359-1362 (2011).

17. J. Cordes et al., "Damage of stone baskets by endourologic lithotripters: a laboratory study of 5 lithotripters and 4 basket types," Adv. Urol. 2013, 1-6 (2013).

18. M. K. Lu et al., "Compact light-emitting diode lighting ring for video-assisted thoracic surgery," J Biomed Opt. 19(10), 105004 (2014).

19. D. M. Wilson, "Clinical and laboratory approaches for evaluation of nephrolithiasis," J. Urol. 141(3 Pt. 2), 770-774 (1989).

20. D. J. Lama et al., "Fluid dynamic analysis of hand-pump infuser and UROMAT endoscopic automatic system for irrigation through a flexible ureteroscope," J. Endourol. 32(5), 431-436 (2018).

21. R. T. Bryan, L. J. Billingham, and D. M. Wallace, "Narrow-band imaging flexible cystoscopy in the detection of recurrent urothelial cancer of the bladder," BJU Int. 101(6), 702-706 (2008).

22. H. W. Herr and S. M. Donat, "A comparison of white-light cystoscopy and narrow-band imaging cystoscopy to detect bladder tumour recurrences," BJU Int. 102(9), 1111-1114 (2008).

23. E. C. Cauberg et al., "A new generation of optical diagnostics for bladder cancer: technology, diagnostic accuracy, and future applications," Eur. Urol. 56(2), 287-297 (2009).

24. T. Inoue et al., "Comparative study of conventional colonoscopy and pan-colonic narrow-band imaging system in the detection of neoplastic colonic polyps: a randomized, controlled trial," J. Gastroenterol. 43(1), 45-50 (2008).

25. P. Sharma et al., "The utility of a novel narrow band imaging endoscopy system in patients with Barrett's esophagus," Gastrointest. Endosc. 64(2), 167-175 (2006).

26. K. Kuznetsov, R. Lambert, and J. F. Rey, "Narrow-band imaging: potential and limitations," Endoscopy 38(1), 76-81 (2006).

27. L. M. Song et al., "Narrow band imaging and multiband imaging," Gastrointest. Endosc. 67(4), 581-589 (2008).

28. Olympus, "NBI for enhancing the visualization of the capillary network and mucosal morphology," http://www.olympus-global.com/ en/technology/technology/luceraelite/.

Christopher R. Wilson is currently a postdoctoral fellow in the Department of Physics and Optical Science, the University of North Carolina at Charlotte.

Joshua D. Kennedy is currently an MS student of applied physics at the University of North Carolina at Charlotte.

Pierce B. Irby is a faculty member in the McKay Department of Urology, Carolinas Medical Center in Charlotte, North Carolina.

Nathaniel M. Fried is a professor in the Department of Physics and Optical Science, the University of North Carolina at Charlotte. He also holds adjunct faculty positions in the McKay Department of Urology, Carolinas Medical Center and the Department of Urology, Johns Hopkins University. His research interests include therapeutic and diagnostic applications of lasers in urology. 